\section{SAT0487 A RANDOMIZED, BLINDED, COMPARATOR-CONTROLLED TRIAL INVESTIGATING A 4-WEEK COURSE OF LYRICA IN SUBJECTS WITH KNEE OSTEOARTHRITIS WHO EXHIBIT NEUROPATHIC PAIN, COMPARED WITH A 4-WEEK COURSE OF PARACETAMOL}

A. Wright ${ }^{1}$, P. Moss ${ }^{1}$, H.A. Benson ${ }^{2}$, R. Will ${ }^{3}$, P. Chowalloor ${ }^{4} .{ }^{1}$ School of Physiotherapy and Exercise Science; ${ }^{2}$ School of Pharmacy, Curtin University; ${ }^{3}$ School of Medicine and Pharmacology, University of Western Australia;

${ }^{4}$ Department of Rheumatology, Royal Perth Hospital, Perth, Australia

Background: Knee osteoarthritis (OA) has been considered the archetypal model of inflammatory or nociceptive pain [1] but it is apparent that people with knee OA may present with different pain phenotypes including some with features of neuropathic pain $[2,3]$.

Objectives: The aim of the present study was to select people with knee OA who exhibited features of neuropathic pain based on elevated cold pain thresholds $\left(\geq 15^{\circ} \mathrm{C}\right)$ and elevated PainDETECT scores $(\geq 13)$ and to determine if pregabalin would be more effective in reducing pain and improving function in this cohort than paracetamol.

Methods: The study used a double-blind, randomized, comparator-controlled design in participants with knee OA to compare the effectiveness of a standard dose of paracetamol (1000mg qid) with a titrated dose of pregabalin (Lyrica) taken daily over 28 days. A cohort of 90 participants with moderate to severe painful knee OA (Pain rating $>4 / 10$ ) who demonstrated features of neuropathic pain were included in the study. Participants were selected from an initial cohort of 271 OA sufferers recruited from the Perth community based on screening of CPT and PainDETECT. Included participants were randomly assigned to receive either a four week course of pregabalin titrated to a maximum dose of $300 \mathrm{mg}$ or a four week course of paracetamol $(4,000 \mathrm{mg})$.

Participants were assessed at baseline, 14 and 28 Days. They completed the PainDETECT, WOMAC and PQAS questionnaires. Quantitative sensory testing was carried out at three sites (index knee, contralateral knee, ECRB) using standard methods. Cold and heat pain thresholds were tested using a Peltier thermode and pressure pain thresholds were assessed using a digital algometer. Physical function was assessed using three timed locomotor function tests (sit-to-stand, walk, stairs).

Results: Participants receiving pregabalin $(300 \mathrm{mg})$ exhibited greater reductions in WOMAC Pain scores $(P=0.0001)$ and PainDETECT scores $(P=0.0001)$ at Day 28 compared to the paracetamol group and a greater increase in pressure pain thresholds (reduced tenderness) $(P=0.025)$ at the index knee. There was no significant difference between the groups in cold pain thresholds $(P=0.33$ ). Participants in the pregabalin group completed the physical tests more quickly than the paracetamol group (Sit-to-stand $p=0.05$, walk $p=0.001$, stairs $p=0.05$ ). The pregabalin group also had a significantly increased likelihood of reducing their PainDETECT score below the entry value of 13 compared to the paracetamol group (OR 9.20; $P=0.001$ ) but there was no significant difference in the likelihood of reducing their cold pain threshold below the $15^{\circ} \mathrm{C}$ entry value (OR 2.26; $\mathrm{P}=0.14)$.

Conclusions: The group receiving pregabalin $300 \mathrm{mg}$ showed greater reductions in pain, reduced features of neuropathic pain and reduced tenderness at the affected knee. Further research is warranted to evaluate pregabalin in this specific patient cohort.

References:

[1] Harden, R.N., et al., J Pain, 2013. 14(3): p. 281-9.

[2] Hochman, J.R., et al., Osteoarthritis Cartilage, 2011. 19(6): p. 647-54.

[3] Ohtori, S., et al., Yonsei Med J, 2012. 53(4): p. 801-5.

Acknowledgements: The investigators thank Ms Lisa Webster for her contribution to the study.

Disclosure of Interest: A. Wright Grant/research support from: Pfizer Inc, P. Moss Grant/research support from: Pfizer Inc, H. Benson Grant/research support from: Pfizer Inc, R. Will Grant/research support from: Pfizer Inc, P. Chowalloor: None declared

DOI: 10.1136/annrheumdis-2017-eular.5656

\section{SAT0488 COMPARATIVE EFFECTIVENESS OF AYURVEDA AND CONVENTIONAL CARE IN KNEE OSTEOARTHRITIS - A RANDOMIZED CONTROLLED TRIAL}

C.S. Kessler ${ }^{1}$, K. Dhiman ${ }^{2}$, A. Kumar ${ }^{3}$, T. Ostermann ${ }^{4}$, S. Gupta ${ }^{5}$, A. Morandi ${ }^{6}$, M. Mittwede ${ }^{7}$, E. Stapelfeldt ${ }^{1}$, M. Spoo ${ }^{1}, K^{2}$ Icke $^{8}$, A. Michalsen ${ }^{1}$, C. Witt ${ }^{8}$. ${ }^{1}$ Complementary and Integrative Medicine, Immanuel Hospital Berlin, Berlin, Germany; ${ }^{2}$ Central Council for Research in Ayurvedic Sciences; ${ }^{3}$ All India Institute of Ayurveda, New Delhi, India; ${ }^{4}$ Department of Psychology and Psychotherapy, University of Witten Herdecke, Witten, Germany; ${ }^{5}$ Department of Kaya Cikitsa, J.S. Ayurveda College \& P.D. Patel Ayurveda Hospital, Nadiad, India; ${ }^{6}$ School of Ayurvedic Medicine, Ayurvedic Point, Milan, Italy; ${ }^{7}$ European Academy of Ayurveda, Birstein; 8 Institute for Social Medicine, Epidemiology and Health Economics, Charité Medical University, Berlin, Germany

Background: Traditional Indian Medicine Ayurveda is used to treat knee osteoarthritis $(\mathrm{OA})$ despite limited evidence.

Objectives: We aimed to evaluate the effectiveness of complex Ayurvedic treatment compared to complex conventional care in knee OA patients.
Methods: According to ACR criteria patients with knee OA were included in a multicenter randomized, controlled trial and treated in 2 hospital outpatient clinics and 2 private outpatient clinics in Germany with 5 physicians and 20 therapists participating. Patients received either Ayurvedic treatment $(n=77)$ or conventional care $(n=74)$ with 15 treatments over 12 weeks. Primary outcome was the change on the Western Ontario and McMaster University Osteoarthritis (WOMAC) Index after 12 weeks (validated German version). Secondary outcomes included the WOMAC subscales; a pain disability index, numeric rating scales for pain and sleep quality, a pain experience scale, a quality-of-life index, a profile of mood index, rescue medication use, and safety issues.

Results: A total of 151 patients (Ayurveda $n=77$, conventional care $n=74$ ) were included. Changes of the WOMAC Index from baseline to 12 weeks were more pronounced in the Ayurveda group (mean difference 61.0 [95\% Cl 52.4;69.6]) than in the conventional group $(32.0[95 \% \mathrm{Cl} 21.4 ; 42.6])$ resulting in a significant difference between groups $(p<0.001)$ and a clinically relevant effect size (Cohen's d 0.68 [95\% Cl 0.35;1.01]). Similar tendencies were observed for all secondary outcomes at week 12. Effects were sustainable at follow-ups after 6 and 12 months.

Conclusions: The results suggest that a complex Ayurvedic treatment might be clinically superior to a complex conventional intervention in the treatment of OA of the knee.

Disclosure of Interest: None declared

DOI: 10.1136/annrheumdis-2017-eular.4692

\section{SAT0489 ASSOCIATION OF CHILDHOOD OVERWEIGHT MEASURES WITH ADULTHOOD KNEE CARTILAGE DEFECTS AND BONE MARROW LESIONS: A 25-YEAR COHORT STUDY}

T. Meng ${ }^{1}$, S. Thayer ${ }^{2}$, A. Venn ${ }^{1}$, F. Cicuttini ${ }^{3}$, L. March ${ }^{4}$, T. Dwyer ${ }^{5}$, A. Halliday ${ }^{2}$, M. Cross ${ }^{4}$, L. Laslett ${ }^{1}$, G. Jones ${ }^{1}$, C. Ding ${ }^{1,3,6}$, B. Antony ${ }^{1}$ ${ }^{1}$ Menzies Institute for Medical Research; ${ }^{2}$ Department of Radiology, Royal Hobart Hospital, Hobart; ${ }^{3}$ Department of Epidemiology and Preventive Medicine Monash University, Melbourne; ${ }^{4}$ Institute of Bone and Joint Research, Sydney;

${ }^{5}$ Murdoch Childrens Research Institute, Melbourne, Australia; ${ }^{6}$ Translational Research Centre, Academy of Orthopaedics, Southern Medical University, Guangzhou, China

Background: Obesity and overweight have been associated with increased risk of knee osteoarthritis (OA). Cartilage defects and bone marrow lesions (BMLs) are imaging biomarkers that predict the development and progression of knee OA. However, there are no studies describing the effect of childhood obesity or overweight on risk of adulthood knee structural abnormalities including cartilage defects and BMLs.

Objectives: To describe associations between body weight, body mass index (BMI), overweight status and fat mass in childhood and knee cartilage defects and BMLs measured using magnetic resonance imaging (MRI) in adulthood 25 years later.

Methods: Subjects broadly representative of the Australian population $(n=327$, aged 31-41 years) were selected from the Australian Schools Health and Fitness Survey of 1985 ( $n=8498$, aged 7-15 years). Childhood measures, including weight, height and skinfolds were measured in 1985. BMI and fat mass were calculated. They underwent T1-weighted fat-suppressed and proton densityweighted fat-suppressed MRI scans 25 years later. Cartilage defects and BMLs were measured from MRI using a semi-quantitative scoring system. Log binomial regression analyses were used to determine the associations of childhood overweight measures with adulthood knee cartilage defects and BMLs.

Results: There were no associations between childhood overweight measures and adulthood tibio-femoral cartilage defects; however, body weight, BMI and overweight status were positively associated with patellar cartilage defects after adjustment for childhood age, duration of follow-up, sex, childhood height (if weight or fat mass was the predictor), childhood injury, adulthood injury and corresponding adulthood measures (RR $1.05,95 \% \mathrm{Cl} 1.01$ to 1.09 for weight; RR $1.10,95 \% \mathrm{Cl} 1.01$ to 1.19 for $\mathrm{BMI}$; RR $2.06,95 \% \mathrm{Cl} 1.19$ to 3.58 for overweight). After further adjustment for patellar BMLs, these associations remained largely unchanged and the association of fat mass with patellar cartilage defects became significant (RR $1.10,95 \% \mathrm{Cl} 1.01$ to 1.21 ).

Childhood overweight measures were not associated with adulthood BMLs in either tibio-femoral or patellar compartments. However, sex-specific analyses showed $\mathrm{BMI}$ and overweight status were associated with higher risks of patellar BMLs after adjustment for covariates in men (RR $1.36,95 \% \mathrm{Cl} 1.03$ to 1.81 for BMI; RR 4.37, 95\% Cl 1.01 to 18.91 for overweight) but not in women.

Conclusions: Childhood overweight measures were significantly associated with increased risk of patellar cartilage defects and BMLs in young adults. In addition, overweight status was significantly associated with patellar BMLs in men which may explain why overweight was only associated with increased knee pain in men as we reported earlier. These results indicate the importance of addressing overweight in childhood to aid in preventing adulthood knee structural abnormalities which increase the risk of OA in later life.

Disclosure of Interest: None declared

DOI: 10.1136/annrheumdis-2017-eular.5668 